\title{
Measurement of cross sections and couplings of the Higgs Bo- son in bosonic decay channels with the ATLAS detector
}

\author{
Nikita Belyaev ${ }^{1}$,a , on behalf of the ATLAS Collaboration \\ ${ }^{1}$ National Research Nuclear University MEPhI (Moscow Engineering Physics Institute), Kashirskoe highway \\ 31, Moscow, 115409, Russia
}

\begin{abstract}
After the discovery of the Higgs boson, the measurement of its properties are of particular importance. In this paper, measurement of the cross sections and couplings of the Higgs boson in bosonic decay channels with the ATLAS detector are presented. Previous measurements of the spin and parity of this new particle, as well as the investigation of its couplings to other SM particles, revealed no significant deviations from the corresponding predictions for the Standard Model Higgs boson. In the years 2015-2017, the centre-of-mass energy $\sqrt{s}$ and the integrated luminosity of the Large Hadron Collider was increased up to $13 \mathrm{TeV}$ and $36.1 \mathrm{fb}^{-1}$, respectively. With this improvements of the LHC, the properties of recently discovered Higgs boson can be studied in more details. In this paper, latest updates on cross sections and couplings analyses of the Higgs Boson are presented. The discussion will focus on the recent results obtained by the ATLAS collaboration in $\gamma \gamma$ and $4 l$ Higgs boson decay channels as well as their combination.
\end{abstract}

\section{Introduction}

The ATLAS and CMS Collaborations at the Large Hadron Collider (LHC) have performed extensive studies of the Higgs-boson properties in the past few years. With the ATLAS detector [1] the Higgsboson mass has been measured to be $m_{H}=125.09 \pm 0.24 \mathrm{GeV}$ [2] and no significant deviations from Standard Model (SM) predictions have been found in the cross sections measured per production mode [3], the branching ratios [4], or spin and parity quantum numbers [5, 6]. Furthermore, inclusive and differential fiducial cross sections of Higgs-boson production, defined as event yields corrected for the detector response, have been measured by the ATLAS and CMS Collaborations in protonproton (pp) collisions at a centre-of-mass energy of $\sqrt{s}=8 \mathrm{TeV}$, using the $4 l(l=e, \mu), \gamma \gamma$ and $e v \mu \nu$ final states [7-10]. These measurements are also consistent with the Standard Model (SM) predictions, motivating higher precision measurements that also probe the kinematic properties of several production processes.

In this paper, fiducial cross sections are presented inclusively for all of the final states of the $H \rightarrow$ $Z Z^{*} \rightarrow 4 l$ decay $(4 e, 4 \mu, 2 e 2 \mu, 2 \mu 2 e)$. Differential fiducial cross sections are presented as functions of kinematic observables that describe Higgs-boson production and decay physics in pp collisions for $H \rightarrow Z Z^{*} \rightarrow 4 l$ and $H \rightarrow \gamma \gamma$ decay channels. They are not split into the two different final states or Higgs-boson production modes, such as gluon fusion ( $\mathrm{ggF}$ ) or vector-boson fusion (VBF). Each of the

\footnotetext{
ae-mail: Nikita.Belyaev@cern.ch
} 
kinematic variables described below is sensitive to different physics effects. For example, the Higgsboson transverse momentum $p_{T}$ can be used to test perturbative QCD calculations, especially when separated into exclusive jet multiplicities. This variable is also sensitive to the Lagrangian structure of the Higgs-boson interactions [11]. The Higgs-boson rapidity distribution $|y|$ is sensitive to the parton distribution functions (PDFs) of the colliding protons. The decay variables $\left|\cos \theta^{*}\right|$ and $m_{34}$ test the spin and parity of the Higgs boson. The variable $\left|\cos \theta^{*}\right|$ is defined as the absolute value of the cosine of the decay angle of the leading lepton pair in the four-lepton rest frame with respect to the beam axis. The variables $m_{12}$ and $m_{34}$ refer to the invariant masses of the leading and subleading lepton pairs and correspond to the invariant mass of the on-shell and off-shell $Z$ bosons produced in the Higgs-boson decay. The number of jets $N_{\text {jets }}$ produced in association with the Higgs boson and the transverse momentum of the leading jet $p_{T}^{\text {lead, jet }}$ both provide sensitivity to the theoretical modeling of high- $p_{T}$ quark and gluon emission. The invariant mass $m_{j j}$ of the two leading jets in the event is sensitive to different production mechanisms. The signed angle between the two leading jets in the transverse plane $\Delta \phi_{j j}$ is another observable that tests the spin and parity nature of the Higgs boson [12].

\section{Measurements in $H \rightarrow Z Z^{*} \rightarrow 4 l$ channel}

In this analysis, Higgs-boson candidates are formed by selecting two same-flavour opposite-sign (SFOS) lepton pairs, which pass the selection criteria (for more details see [13]). The analysis selection proceeds in parallel for the four final states $(4 \mu, 2 e 2 \mu, 2 \mu 2 e, 4 e)$. The invariant mass distribution of the four leptons of the selected events is shown in Figure 1. Only events with a four-lepton invariant mass in the range $115-130 \mathrm{GeV}$ are used in the final fit.

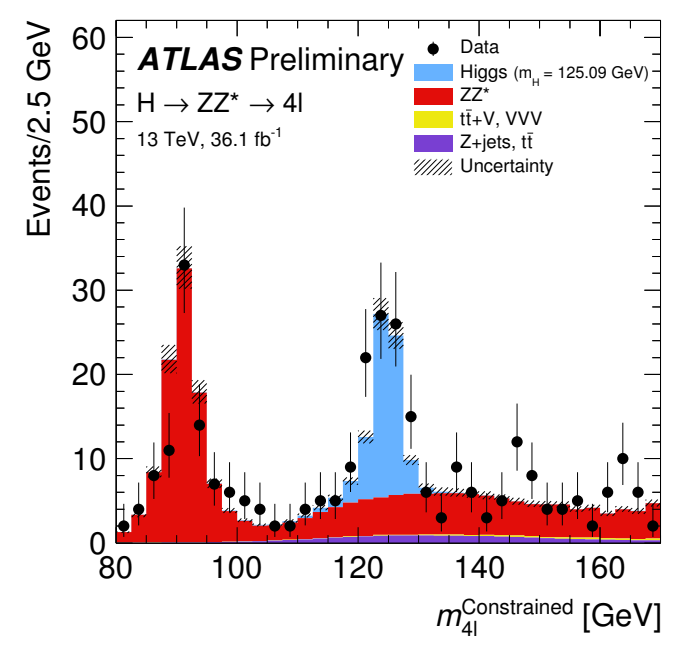

Figure 1. The observed and expected four-lepton invariant mass distribution for the selected Higgs boson candidates with a constrained $Z$ boson mass, shown for an integrated luminosity of $36.1 \mathrm{fb}^{-1}$ and at $\sqrt{s}=13 \mathrm{TeV}$ assuming the SM Higgs boson signal with a mass $m_{H}=125.09 \mathrm{GeV}$. The error bars represent $68.3 \%$ central confidence interval statistical uncertainty. The systematic uncertainty on the prediction is shown by the dashed band [14].

The Higgs boson couplings to SM vector bosons and gluons are studied by measuring the cross sections for different production modes and by probing BSM contributions in tensor couplings. In both approaches, the reconstructed Higgs boson candidate events are classified into different categories. The categories are defined to be sensitive to different Higgs boson production modes, which in turn also provides sensitivity to the beyond the Standard Model (BSM) contributions. The event yields in each category serve as the final discriminant for both the cross section and the tensor structure studies. For the cross section measurement there are also additional discriminant observables introduced in 
reconstructed categories with a sufficiently high number of events. These observables are constructed using dedicated boosted decision trees (BDTs) [15].
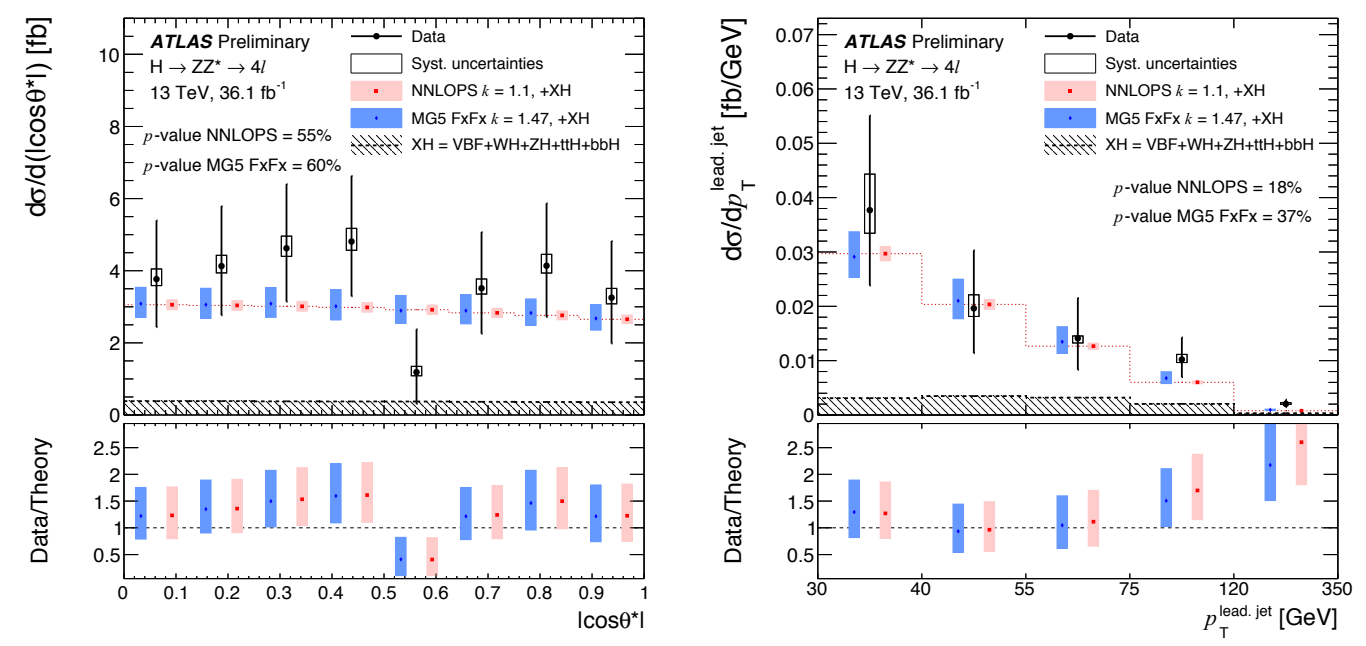

Figure 2. (Left) Differential fiducial cross sectionsas function of the cosine of the decay angle of the leading lepton pair in the four-lepton rest frame with respect to the beam axis $\left|\cos \left(\theta^{*}\right)\right|$. (Right) Differential fiducial cross sectionsas function of the transverse momentum of the leading jet $p_{T}^{\text {lead.jet }}$ [13].

The study of the coupling properties of the Higgs boson have been performed by using the LHC pp collision data, collected by the ATLAS experiment. The Higgs boson candidate events are categorised into several topologies, providing sensitivity to different production modes in various regions of the phase space. BDT discriminants [15] are used to further improve the sensitivity in reconstructed categories with sufficiently large number of events. Figure 2 demonstrates measured differential fiducial cross sections as a function of $\left|\cos \left(\theta^{*}\right)\right|$ and $p_{T}^{\text {lead.jet }}$. These cross sections are compared to ggF predictions by NNLOPS, MG5_aMC@NLO with FxFx jet merging, all normalized to the N3LO cross section with the listed $k$-factors. Predictions for all other Higgs production modes XH are added. The error bars on the data points show the total uncertainties, while the systematic uncertainties are indicated by the boxes. The measured cross sections as function of $\left|\cos \left(\theta^{*}\right)\right|$ are slightly higher than the predictions, but the distribution is consistent with the SM predictions within the uncertainties. The good agreement between data and prediction of the cross sections as a function of $p_{T}^{\text {lead.jet }}$ was observed. All of the measured distributions are consistent with dedicated measurements that have shown the Higgs boson to have the spin and parity as predicted by the SM.

Figure 3 (left) contains $68 \%$ and $95 \% \mathrm{CL}$ two-dimensional contours at $\left(\sigma_{g g H} \cdot B R, \sigma_{V B F} \cdot B R\right)$ plane, which corresponds to $1 \sigma$ and $2 \sigma$ deviation from the measured best fit value, respectively. The largest deviation of $2.2 \sigma$ from the Standard Model is observed for the VBF production. This excess appears due to an observed excess of events characterized by the presence of at least two jets and a dijet invariant mass above $120 \mathrm{GeV}$. The inclusive cross section in the Higgs boson rapidity range of $\left|y_{H}\right|<2.5$ is measured to be $\sigma \cdot \mathrm{BR}\left(H \rightarrow Z Z^{*}\right)=1.73_{-0.24}^{+0.26}$ pb relative to the SM prediction of $1.34 \pm 0.06 \mathrm{pb}$.

Results are also interpreted in terms of $\kappa$ couplings (so-called $\kappa$-framework) $[16,17]$ with coupling modifiers $\kappa_{V}$ and $\kappa_{f}$, showing consistency with the SM (Figure 3, right). Based on event yields 

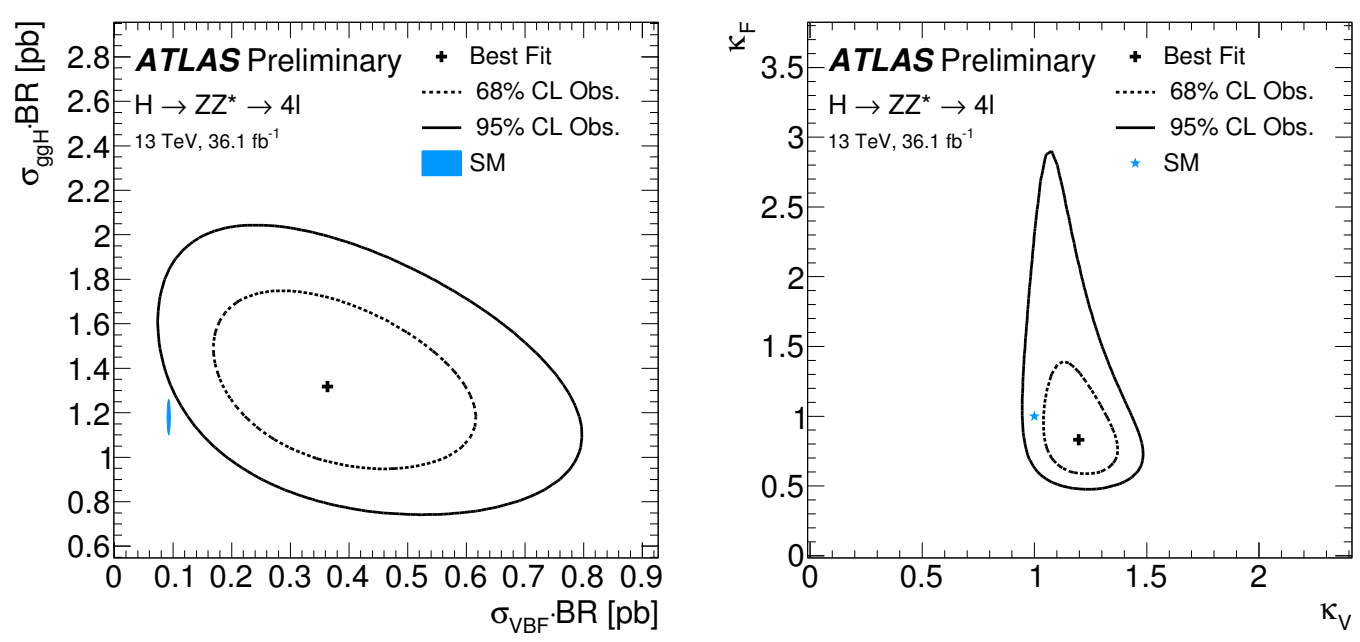

Figure 3. (Left) Likelihood contours at $68 \% \mathrm{CL}$ (dashed line) and $95 \% \mathrm{CL}$ (solid line) in the $\left(\sigma_{g g H} \cdot B R\right.$, $\sigma_{V B F} \cdot B R$ ) plane and (right) likelihood contours in the $\kappa_{V}-\kappa_{f}$ plane. The best fits to the data (solid cross) and the SM expectations are also indicated. In (left), the SM expectation is shown together with its theory uncertainty (filled blue elipse), while in (right) only the central value of the SM expectation (solid blue star) is shown [14].

observed in each reconstructed event category, constraints are placed on possible BSM interactions of the Higgs boson within the framework of an effective Lagrangian extension of the SM. The data are shown to be consistent with the SM hypothesis, with the largest deviations of about $1.5 \sigma$ due to the mentioned excess of observed events. Exclusion limits are set on the CP-even and CP-odd BSM couplings of the Higgs boson to vector bosons and on the CP-odd BSM Higgs boson coupling to gluons and shown at table 1.

Table 1. Observed and expected limits at $95 \% \mathrm{CL}$ on $k_{A g g}, k_{H V V}$ and $k_{A V V}$ coupling parameters, their best-fit values and corresponding compatibility with the SM expectation, as obtained from the negative log-likelihood scans. The coupling $k_{H g g}$ is fixed to the SM value of 1 in the fit, while the coupling $k_{S M}$ is either fixed to the SM value of 1 or left as a free parameter of the fit [14].

\begin{tabular}{ccccccc}
\hline $\begin{array}{c}\text { BSM coupling } \\
k_{B S M}\end{array}$ & $\begin{array}{c}\text { Fit } \\
\text { configuration }\end{array}$ & $\begin{array}{c}\text { Expected } \\
\text { limit }\end{array}$ & $\begin{array}{c}\text { Observed } \\
\text { limit }\end{array}$ & $\begin{array}{c}\text { Best-fit } \\
\hat{k}_{B S M}\end{array}$ & $\begin{array}{c}\text { Best-fit } \\
\hat{k}_{S M}\end{array}$ & $\begin{array}{c}\text { Deviation } \\
\text { from SM }\end{array}$ \\
\hline$k_{A g g}$ & $\left(k_{H g g}=1, k_{S M}=1\right)$ & {$[-0.47,0.47]$} & {$[-0.68,0.68]$} & \pm 0.43 & - & $1.8 \sigma$ \\
$k_{H V V}$ & $\left(k_{H g g}=1, k_{S M}=1\right)$ & {$[-2.9,3.2]$} & {$[0.8,4.5]$} & 2.9 & - & $2.3 \sigma$ \\
$k_{H V V}$ & $\left(k_{H g g}=1, k_{S M}\right.$ free $)$ & {$[-3.1,4.0]$} & {$[-0.6,4.2]$} & 2.2 & 1.2 & $1.7 \sigma$ \\
$k_{A V V}$ & $\left(k_{H g g}=1, k_{S M}=1\right)$ & {$[-3.5,3.5]$} & {$[-5.2,5.2]$} & \pm 2.9 & - & $1.4 \sigma$ \\
$k_{A V V}$ & $\left(k_{H g g}=1, k_{S M}\right.$ free $)$ & {$[-4.0,4.0]$} & {$[-4.4,4.4]$} & \pm 1.5 & 1.2 & $0.5 \sigma$ \\
\hline
\end{tabular}

\section{Measurements in $H \rightarrow \gamma \gamma$ channel}

Properties of the Higgs boson are also measured in the two-photon final state using proton-proton collision data. The ATLAS and CMS mass measurement of $125.09 \pm 0.24 \mathrm{GeV}$ is used and signals 
are extracted in a diphoton mass range of $105-160 \mathrm{GeV}$. Production cross section measurements for a Higgs boson rapidity $\left|y_{H}\right|<2.5$ and in the full phase space for gluon fusion $(\mathrm{ggH})$, vector boson fusion (VBF), and Higgs boson production in association with a vector boson or a top-quark pair are obtained. The signal strength, defined as the ratio of the observed signal yield to the expected signal yield, is measured for the same production processes, as well as inclusively. The fitted invariant mass spectra for all regions was calculated. The result of signal-plus-background fit to this spectrum is shown in Figure 4.
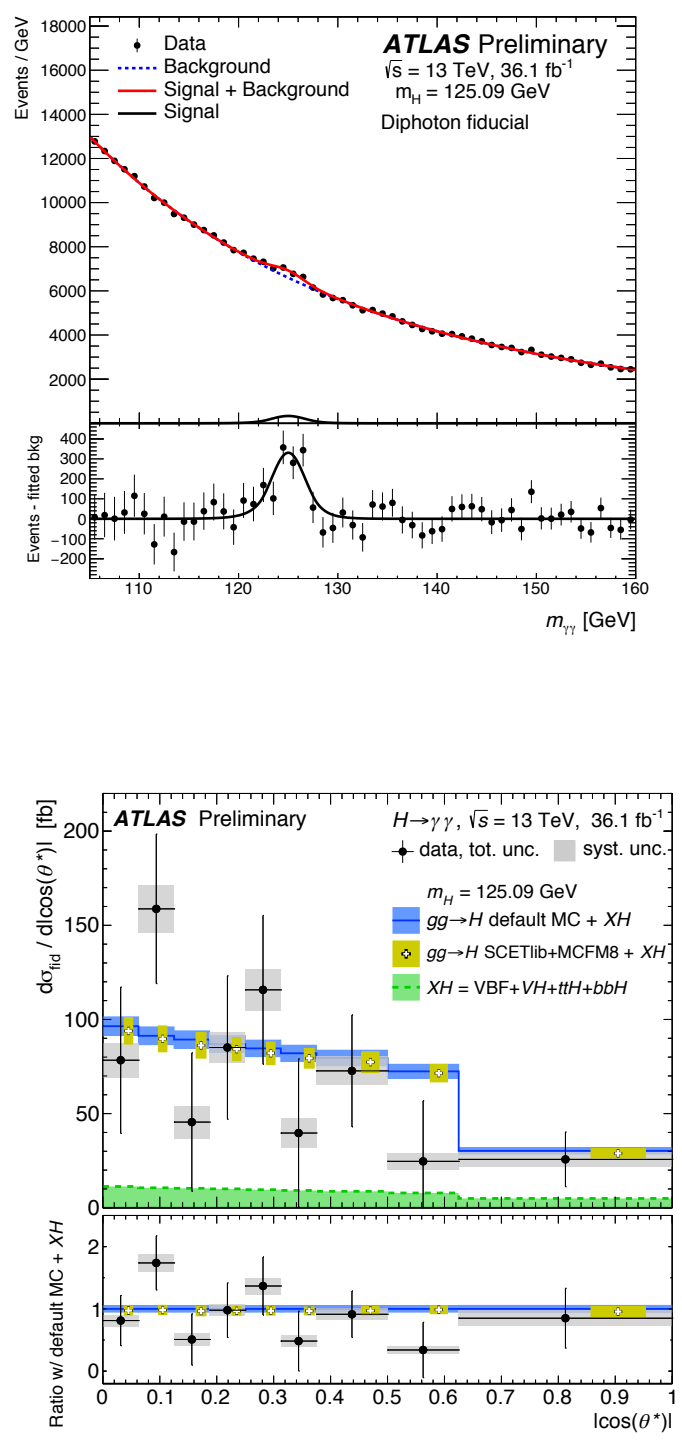

Figure 4. Diphoton invariant mass $m_{\gamma \gamma}$ spectrum observed in the $13 \mathrm{TeV}$ data from 2015 and 2016 data is shown for the diphoton fiducial region. The solid red curve shows the fitted signal-plus-background model when the Higgs boson mass is constrained to be $125.09 \pm 0.24 \mathrm{GeV}$. The background component of the fit is shown with the dotted blue curve. The signal component of the fit is shown with the solid black curve. The bottom plot shows the residuals between the data and the background component of the fitted model [18].

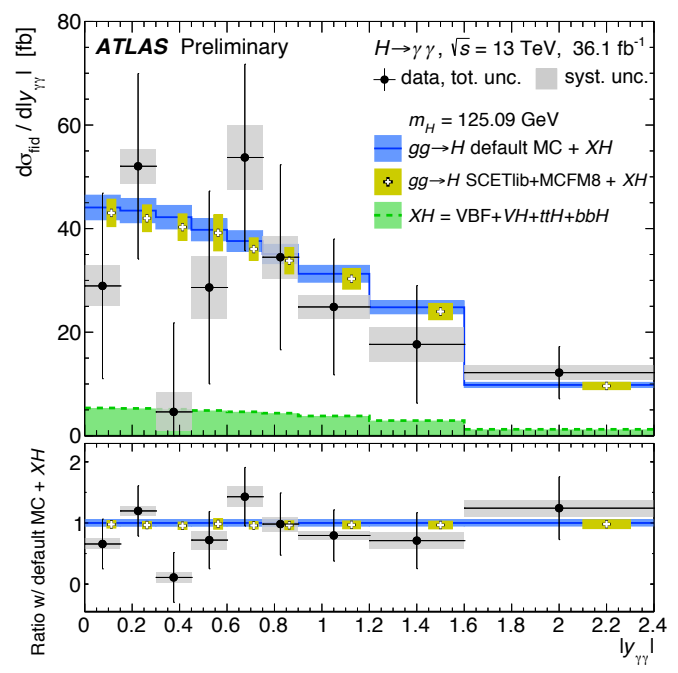

Figure 5. The differential cross section for $p p \rightarrow H \rightarrow \gamma \gamma$ as a function of $\left|\cos \left(\theta^{*}\right)\right|$ (left) and $\left|y_{\gamma \gamma}\right|$ (right) are shown and compared to the SM expectation [18]. 
Differential cross sections dominated by ggH production are measured as a function of diphoton kinematic variables sensitive to quantum chromodynamics and the spin of the Higgs boson. Measured differential cross sections are shown at Figure 5. The data are shown as filled (black) circles. The vertical error bar on each data point represents the total uncertainty in the measured cross section and the shaded (grey) band is the systematic component. The SM prediction, defined using the Powheg NNLOPS prediction for gluon fusion and the default MC samples for the other production mechanisms, is presented as hatched (blue) band, with the depth of the band reflecting the total theoretical uncertainty (see text for details). The small contribution from $V B F, V H, t t H$ and $b b H$ is also shown as a (green) histogram and denoted by $X H$. These are compared with state-of-the-art Standard Model predictions, where no significant deviations are observed.
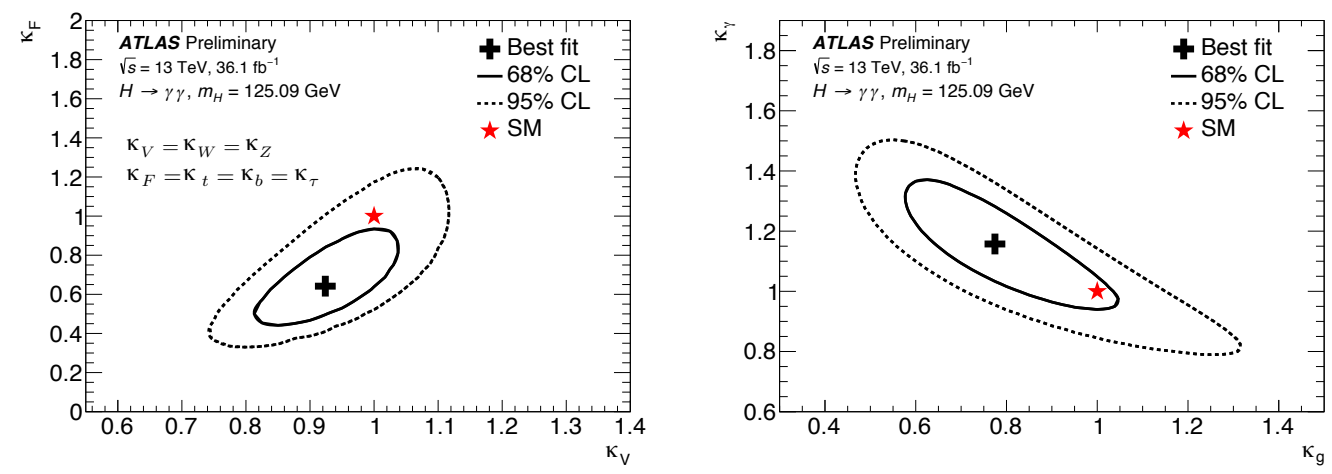

Figure 6. Likelihood contours in (left) the $\left(\kappa_{g}, \kappa_{\gamma}\right)$ plane, and (right) the $\left(\kappa_{V}, \kappa_{f}\right)$ plane, compared to the Standard Model prediction (red star) for a Higgs boson mass $m_{H}=125.09 \mathrm{GeV}$. In (left), the coupling strength parameters $\kappa_{f}$ and $\kappa_{V}$ are assumed to be equal to one. In (right), the coupling strength parameters $\kappa_{g}$ and $\kappa_{\gamma}$ are treated as a function of the more fundamental coupling-strength parameters $\kappa_{f}$ and $\kappa_{V}$ for the particles that contribute to the SM loop processes inducing the Higgs boson couplings to gluons and photons [18].

The $68 \%$ and $95 \%$ CL two-dimensional contours of the effective couplings $\kappa_{g}$ and $\kappa_{\gamma}$ are shown in Figure 6 (left), where the $\kappa_{V}$ and $\kappa_{f}$ parameters affecting other production modes are fixed to the $\mathrm{SM}$ assumption. For the second model, the ggH and $H \rightarrow \gamma \gamma$ loops are resolved in terms of $\kappa_{V}$ and $\kappa_{f}$. The $68 \%$ and 95\% CL two-dimensional contours of $\kappa_{f}$ and $\kappa_{V}$ are shown in Figure 6 (right). The $\mathrm{SM}$ prediction is found within the $68 \% \mathrm{CL}$ contour for the former scenario and within the $95 \% \mathrm{CL}$ contour for the latter one.

The signal strength measurements for the same production processes as well as globally are carried out and shown at Figure 7. The global signal strength measurement of $0.99 \pm 0.14$ improves on the precision of the previous ATLAS measurement by a factor of 2, and $\mathrm{ggH}$ (VBF) production is measured to be $1 \sigma$ below ( $2 \sigma$ above) the Standard Model expectation. The fiducial cross section is measured to be $\sigma_{\text {fid }}=54.7 \pm 9.1$ (stat.) \pm 4.5 (syst.) $\mathrm{fb}$, for a Higgs boson decaying into two isolated photons that have transverse momentum greater than $35 \%$ and $25 \%$ of the diphoton invariant mass and each with absolute pseudorapidity $|\eta|<2.37$, excluding the region $1.37<|\eta|<1.52$. The Standard Model prediction for the same fiducial region is $63.5 \pm 2.4 \mathrm{fb}$. No significant deviations from the Standard Model expectations are observed. All reported results are statistically limited and their precision will further improve with the full data set to be recorded during Run 2 of the LHC. 


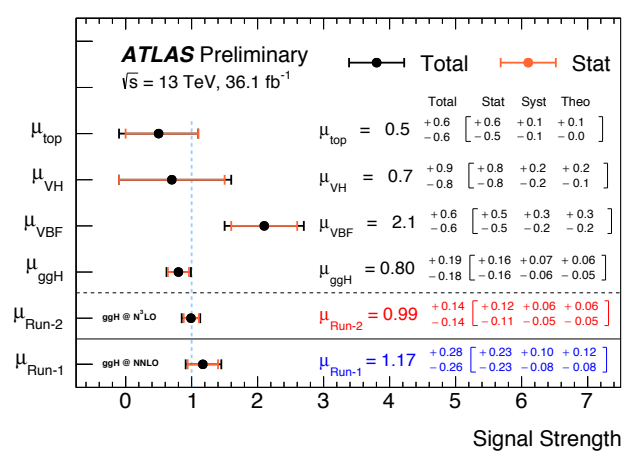

Figure 7. Summary of the signal strengths measured for the different production processes $(g g H, V B F$, $V H$ and $t t H+t H)$ and globally $\left(\mu_{R u n-2}\right)$, compared to the global signal strength measured at 7 and $8 \mathrm{TeV}$ $\left(\mu_{\text {Run-1 }}\right)$ [19]. The black (red) error bar shows the total (statistical) uncertainty. The $\left(\mu_{R u n-1}\right)$ was derived assuming the Higgs production cross section based on Ref. [16, 20]. In the more recent theoretical predictions used in this analysis $[17,21]$, the gluon fusion production cross section is larger by approximately $10 \%[18]$.

\section{Combined measurements in $H \rightarrow Z Z^{*} \rightarrow 4 l$ and $H \rightarrow \gamma \gamma$ channels}

Measurements of the Higgs boson production cross sections and branching ratios have been performed by using $\sqrt{s}=13 \mathrm{TeV}$ proton-proton collision data. The combination of the $4 l$ and $\gamma \gamma$ Higgs boson decay channels allows to maximize the precision of these measurements compared to separate channels. The results of production cross sections measurements are shown at Figure 8.

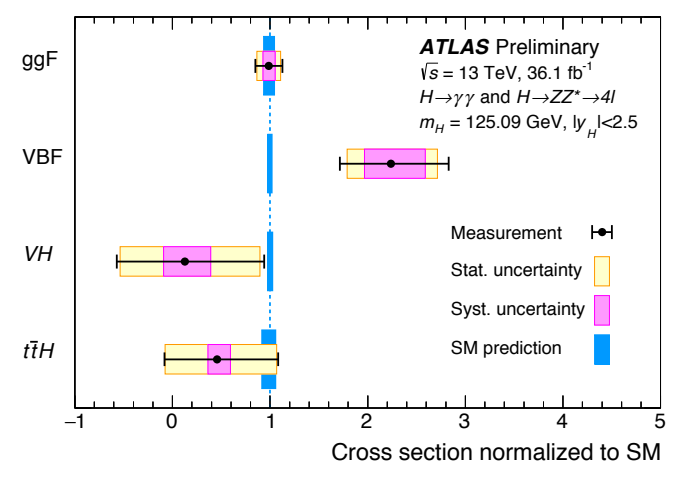

Figure 8. Cross sections for $g g F, V B F, V H$, and $\bar{t} H$ normalized to the SM predictions and measured with the assumption of SM branching fractions. The black error bars and pink and yellow boxes show the total, systematic, and statistical uncertainties in the measurements, respectively. The blue bands indicate the theoretical uncertainties in the predictions [22].

An inclusive measurement of the signal yield is translated into the total Higgs boson production cross section. The cross section of $p p \rightarrow H+X$ at $\sqrt{s}=13 \mathrm{TeV}$ is measured to be $57.0_{-5.9}^{+6.0}$ (stat.) $)_{-3.3}^{+4.0}$ (syst.) pb, consistent with the SM prediction of $55.6_{-3.4}^{+2.4} \mathrm{pb}$. In addition, the global signal strength is determined by dividing the data into individual measurement categories, with the result $\mu=\sigma \cdot B r /(\sigma \cdot B r)_{S M}=1.09 \pm 0.12$. The Higgs boson production cross sections are measured in $\left|y_{H}\right|<2.5$ in a combined fit for each of the processes $g g F, V B F, V H$, and $t t H$, assuming the SM Higgs branching fractions to $\gamma \gamma$ and $Z Z^{*} \rightarrow 4 l$. Removing the SM assumption on branching fractions, a fit is performed for $\sigma_{g g F} \cdot B r_{4 l}, \sigma_{\text {prod }} / \sigma_{g g F}$, and $B r_{\gamma \gamma} / B r_{4 l}$. The processes are further divided into kinematic regions and combined measurements of the cross sections and the ratio of branching fractions are performed in these regions. Finally, the observed Higgs boson yields are used to obtain confidence intervals for $\kappa$ modifiers to the couplings of the SM Higgs boson to fermions, weak vector bosons, gluons, and photons. No significant deviation from the Standard Model predictions is observed.

The individual channel measurements are combined to obtain confidence intervals for the $k_{i}$ coefficients using progressively relaxed assumptions on the coupling relationships. First, two-parameter fits are performed for the coefficients of couplings to fermions $\left(k_{f}\right)$ and to weak vector bosons $\left(k_{V}\right)$, 

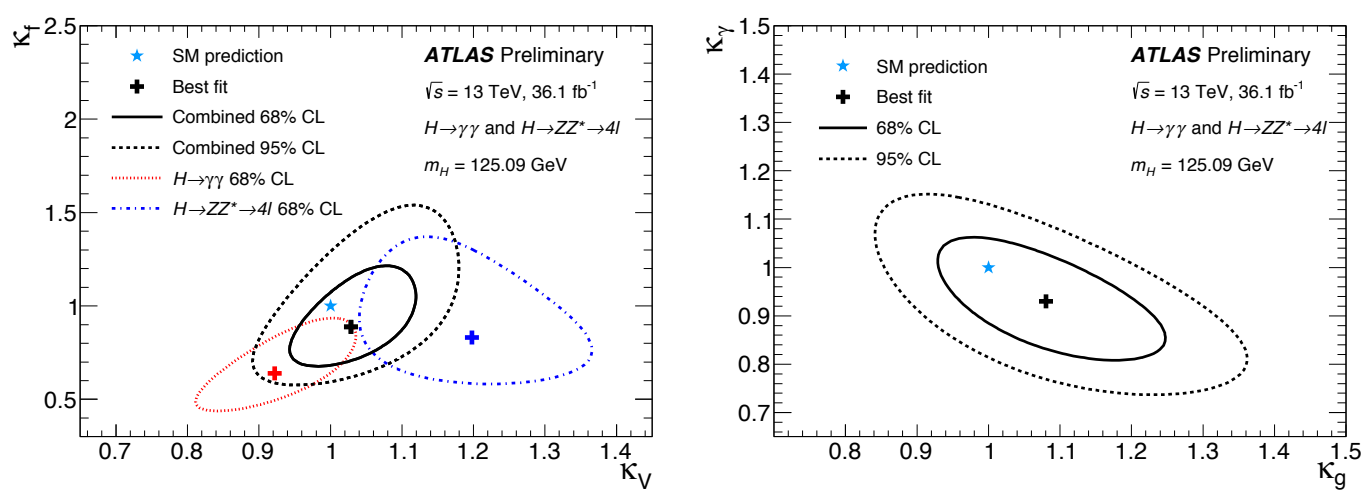

Figure 9. (Left) Contours at $68 \%$ and $95 \% \mathrm{CL}$ in the $\left(\kappa_{f}, \kappa_{V}\right)$ plane. (Right) Contours at $68 \%$ and $95 \%$ CL in the $\left(\kappa_{\gamma}, \kappa_{g}\right)$ plane [22].

and for the coefficients $k_{g}$ and $k_{\gamma}$. In these fits the branching fraction to non-SM particles is assumed to be zero. This assumption is removed in a four-parameter fit, where the coefficient for the Higgs boson width $k_{H}$ is added to a combination of ratios of the other $k$ parameters. The fits assume no other deviations from the SM and include theory uncertainties on the predicted signal yields, as in the case of the signal strength measurement.

The dominant production mechanisms of ggF and VBF have $k_{f}^{2}$ and $k_{V}^{2}$ dependencies, respectively. The fit results are summarized in Figure 9 (left) and show a small positive correlation due in part to the destructive interference between the top-quark and W-boson loops in the $H \rightarrow \gamma \gamma$ decay. The two-parameter fit for $k_{g}$ and $k_{\gamma}$ (Figure 9, right) shows a strong anti-correlation because the leading constraint comes from $H \rightarrow \gamma \gamma$ in the gluon fusion channel.

The ggF and VBF cross sections are measured with the best precision, though the results are anticorrelated because the VBF selection categories have significant contributions from ggF production. Figure 10 shows the measured likelihood contours in the $\sigma_{V B F}$ versus $\sigma_{g g F}$ plane from $H \rightarrow \gamma \gamma$ and $H \rightarrow Z Z^{*} \rightarrow 4 l$, and their combination. The cross sections for $\mathrm{VH}$ and $\mathrm{ttH}$ are profiled with the data. The two-dimensional compatibility between the measurement and the SM prediction corresponds to a p-value of $p_{S M}=3 \%$.

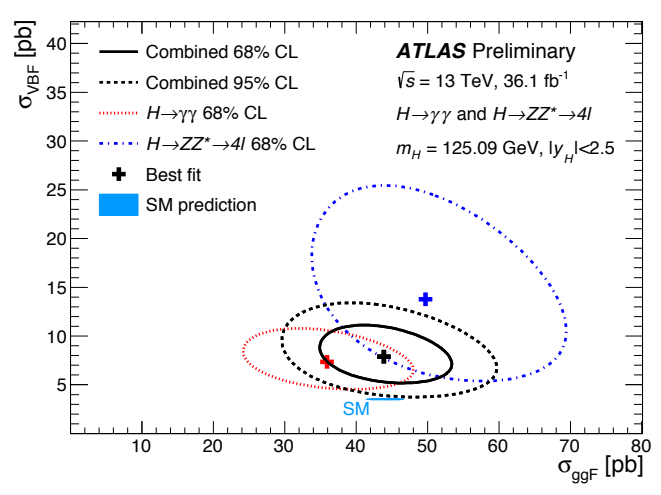

Figure 10. Contours in the $\sigma_{V B F}$ versus $\sigma_{g g F}$ plane as measured in $H \rightarrow \gamma \gamma$ (red) and $H \rightarrow Z Z^{*} \rightarrow 4 l$ (dark blue), as well as their combination (black), together with the SM prediction (light blue). In the combination the branching fractions are fixed to their SM values [22]. 
A set of four ratios is constructed to probe the loop vertices $\left(k_{g}, k_{\gamma}\right)$, total width $\left(k_{H}\right)$, and the fermion and vector couplings ( $k_{f}$ and $k_{V}$ respectively): $k_{g V}=k_{g} k_{f} / k_{H}, \Lambda_{V g}=k_{V} / k_{g}, \Lambda_{f g}=k_{f} / k_{g}$, and $\Lambda_{\gamma V}=k_{\gamma} / k_{V}$. The parameter $\Lambda_{f g}$ is allowed to go negative to exploit the moderate sensitivity to then relative sign from $t H X$ and $g g \rightarrow Z H$ processes. All the other parameters are assumed to be positive without losing generality. The inclusion of $k_{H}$ in the parameterization allows for non-SM decays of the Higgs boson, but this parameter is not determined directly. The results are shown in Figure 11. The four-dimensional compatibility with the SM prediction is $p_{S M}=15 \%$.

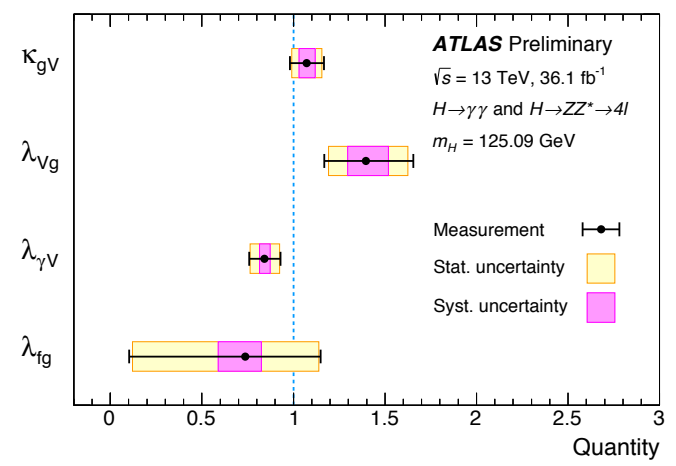

Figure 11. Best-fit values and uncertainties for $\kappa_{g V}$, $\Lambda_{V g}, \Lambda_{\gamma V}$, and $\Lambda_{f g}$. The black, pink, and yellow error bars show the total, systematic, and statistical uncertainties, respectively [22].

The results of mass measurements from each of the individual channels and their combination, along with the LHC Run 1 result, are summarized in Figure 12. The systematic (magenta-shaded bands), statistical (yellow-shaded bands), and total (black error bars) uncertainties are indicated. The (red) vertical line and corresponding (gray) shaded column indicate the central value and the total uncertainty of the combined measurement, respectively.

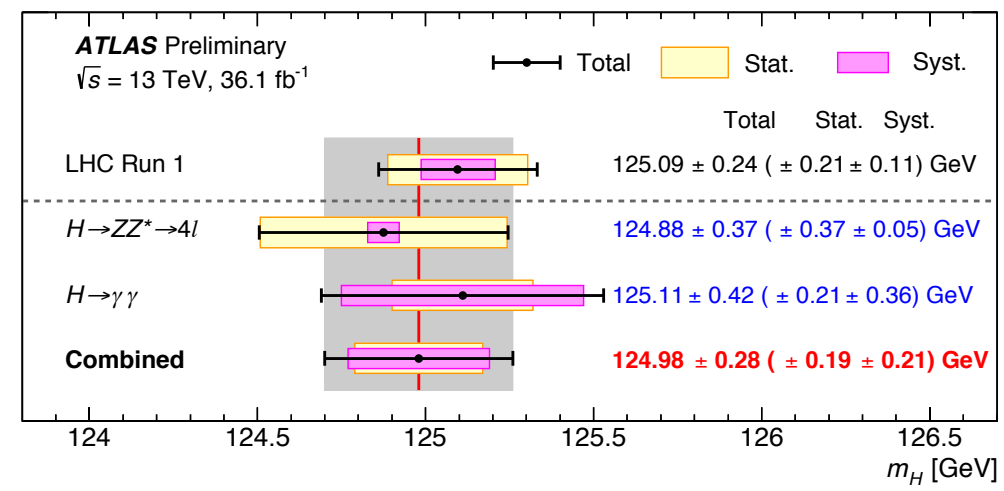

Figure 12. Summary of the Higgs boson mass measurements from the individual and combined analyses [23] performed here, compared to the combined Run 1 measurement by ATLAS and CMS [2]. 


\section{Conclusion}

The review of ATLAS collaboration analyses was presented related to $H \rightarrow Z Z^{*} \rightarrow 4 l, H \rightarrow \gamma \gamma$ channels and their combination. Results of these analyses include measured differential and fiducial cross sections, limits on coupling constants and the updated value of the Higgs boson mass. No deviations from the Standard Model were observed in bosonic decay channels of the Higgs boson. Full review of presented analyses and all the technical information can be found at the corresponding conference notes $[13,14,18,22,23]$.

\section{Acknowledgments}

We gratefully acknowledge the financial support from Russian Science Foundation - grant No.17-7210021.

\section{References}

[1] ATLAS Collaborations, JINST 3, S08003 (2008).

[2] ATLAS and CMS Collaborations, Phys. Rev. Lett. 114, 191803 (2015).

[3] ATLAS Collaboration, Eur. Phys. J. C 76, 6 (2016).

[4] ATLAS and CMS Collaborations, JHEP 08, 045 (2016).

[5] ATLAS Collaboration, Phys. Lett. B 726, 120 (2013).

[6] ATLAS Collaboration, Phys. J. C 114, 191803 (2015).

[7] ATLAS Collaboration, Phys. Lett. B 738, 234 (2014).

[8] ATLAS Collaboration, JHEP 09, 112 (2014).

[9] ATLAS Collaboration, Phys. Rev. Lett. 115, 091801 (2015).

[10] ATLAS Collaboration, JHEP 08, 104 (2016).

[11] M. Grazzini, A. Ilnicka, M. Spira and M. Wiesemann, JHEP 03, 115 (2017).

[12] J. M. Campbell, R. K. Ellis and G. Zanderighi, JHEP 10, 028 (2006).

[13] ATLAS Collaboration, ATLAS-CONF-2017-032 (2017).

[14] ATLAS Collaboration, ATLAS-CONF-2017-043 (2017).

[15] M. Bramer, Principles of Data Mining, (Springer, London, 2007) 343.

[16] LHC Higgs Cross Section Working Group (S. Heinemeyer et al.), Handbook of LHC Higgs Cross Sections: 3. Higgs Properties, (CERN, Geneve, 2013) 404.

[17] LHC Higgs Cross Section Working Group (D. de Florian et al.), Handbook of LHC Higgs Cross Sections: 4. Deciphering the Nature of the Higgs Sector, (CERN, Geneve, 2016) 868.

[18] ATLAS Collaboration, ATLAS-CONF-2017-045 (2017).

[19] ATLAS Collaboration, Phys. Rev. D 90, 112015 (2014).

[20] G. D’Agostini, Nucl. Instrum. Meth. A 362, 487 (1995).

[21] A. Hocker and V. Kartvelishvili, Nucl. Instrum. Meth. A 372, 469 (1996).

[22] ATLAS Collaboration, ATLAS-CONF-2017-047 (2017).

[23] ATLAS Collaboration, ATLAS-CONF-2017-046 (2017). 